\title{
A Novel BCAA-catabolism Related Gene Signature for Overall Survival Prediction of Pancreatic Carcinoma
}

\section{Ziting Jiang}

Fudan University Shanghai Cancer Center

Jiajie Zheng

Fudan University Shanghai Cancer Center

Jianqiang Liu

Fudan University Shanghai Cancer Center

Xiujiang Yang

Fudan University Shanghai Cancer Center

Ke Chen ( $\square$ chenke_1990@126.com )

Fudan University Shanghai Cancer Center https://orcid.org/0000-0002-3070-7926

\section{Research}

Keywords: Pancreas, Macrophage, Branched-chain amino acid, Enzyme, Prognosis

Posted Date: July 22nd, 2021

DOI: https://doi.org/10.21203/rs.3.rs-721731/v1

License: (c) (i) This work is licensed under a Creative Commons Attribution 4.0 International License. Read Full License 


\section{Abstract \\ Background}

Branched-chain amino acid (BCAA) metabolism plays an important role in the pancreatic carcinogenesis, but its mechanism remains unclear. Hence, this study was performed to investigate comprehensively the value of genes related to BCAA catabolism in pancreatic cancer.

\section{Methods}

The online GEO, TCGA, and ICGC datasets were searched for bioinformatic analysis. Univariate Cox and Lasso regression were applied to construct a predictive model. Human cancer cell lines and tissue microarray (TMA) were applied for validation.

\section{Results}

From the 48 BCAA-catabolism enzyme (BCE) genes, a 5-gene risk-score (ABAT, ACAT1, BCAT1, BCAT2, and DBT) was constructed. Patients in high-risk and low-risk groups stratified by risk-score, indicated significantly different OS (for TCGA, 1.62 years vs. 1.90 years, $p=0.02$; for ICGC, 1.25 years vs. 1.87 years, $p=0.01$; for GEO, 1.49 years vs. 2.63 years, $p=0.01$ ). Given the clinical parameters, the risk-score was independent predictor for prognosis [for TCGA, hazard ratio $(\mathrm{HR})=3.16,95 \%$ confidence interval $(\mathrm{Cl})=$ 1.63-6.12, $p<0.01$; for ICGC, HR = 3.16,95\% Cl=1.04-2.35, $\mathrm{p}=0.03$ ]. Among the five genes, ABAT and BCAT2 were hub genes with favorable prognosis value, which validated by TMA immunohistochemistry (IHC) staining. Immune infiltration analysis indicated high-risk group enriched macrophage, and decreased positive cell density of stromal $\mathrm{CD} 68^{+}$macrophage in TMA was observed for BCAT2 with lowexpression than high-expression cases $(p<0.05)$.

\section{Conclusion}

A risk-score involving five $\mathrm{BCE}$ genes was proposed to predict the poor prognosis of pancreatic cancer. Based on the immune infiltration analysis, the underlying mechanism might be BCAT2 associated stromal macrophage infiltration.

\section{Introduction}

According to the 2021 Cancer Statistics, pancreatic cancer remains as one of the top ten most common cancers and the fourth leading cause of cancer death worldwide.(1) Two major obstacles for the improvement of prognosis are the diagnosis of early-stage resectable cancer and response rate for therapy. Resistance against first-line chemotherapy treatments always induces the progression and 
recurrence of tumors. Hence, identifying patients with poor prognosis is critical for the management of pancreatic cancer.

Cancer harbors a reprogrammed metabolic profile to meet the energetic needs of the aberrant and neoplastic regeneration.(2) The pancreas is the foremost exocrine and endocrine organ, and its metabolic function is speculated to be critical in carcinogenesis. Hence, the metabolism of branched-chain amino acid (BCAA) has been paid great attention. Numerous studies have demonstrated that BCAA metabolism is influenced by cancer, to a great extent. (3) Two recent prospective cohort studies have indicated that the serum BCAA level in the peripheral blood is a high-risk factor for the development of pancreatic cancer.(4-6) However, the exact mechanism of BCAA in carcinogenesis is unclear. In the human body, 40 BCAA-catabolism enzymes (BCE) have been identified, some of which, such as the branched-chain aminotransferase (BCAT), have emerged as useful markers for tumor prognosis.(3) Whether the BCEs are correlated with the prognosis of pancreatic cancer remains largely unknown.

The tumor infiltration immune cells are important components in the tumor microenvironment, which plays a pivotal role during the initiation, progression, and metastasis of tumors. Macrophage is one of the earliest infiltrating immune cells in pancreatic cancer, and associated with tumor progression and metastasis.(7) Silva et al. reported the immunomodulatory function of BCAA catabolism in glioblastoma by reducing the phagocytic activity of macrophages.(8) Recently study also indicated role of BCAT1 in pro-inflammatory macrophage activation.(9) But the detailed association between BCAA metabolism and macrophage was still unknown in pancreatic cancer.

The genomic landscapes of pancreatic cancer have been well-described and studied. $(10,11)$ The representative sequencing databases are Gene Expression Omnibus database (GEO), The Cancer Genome Atlas (TCGA), and International Cancer Genome Consortium (ICGC). To elucidate the mechanism of the BCAA metabolic enzymes, the current study applied three online databases to investigative role of $B C E$ genes and construct model for prognosis prediction. Further, the bioinformatic findings were validated in human cancer cell lines and tissue microarray (TMA) of human pancreatic cancer. Immune infiltration analysis was also performed to check the relationship between BCE genes and macrophage.

\section{Material And Methods}

\section{Data sources and processing}

To identified the prognosis related genes, genomic data of RNA sequence and micro-array for pancreatic cancer were queried from TCGA cohort, ICGC cohort, and GEO cohort on Sep 1, 2020. PKM value of TCGA cohort, normalized counts value of ICGC cohort, and RAM value of GEO cohort were extracted and applied for further analysis. Survival data was available for TCGA cohort, ICGC cohort, and one GEO cohort (GSE57495). To investigate the expression level of BCE genes in pancreatic cancer, three GEO databases (GSE15471, GSE16515, and GSE28735) which included both tumor and normal control samples. Totally, 48 BCE genes were retrieved from literature and KEGG database (Supplemental Table 1).(10) This is a secondary analysis based on the open online database, and the informed consent was waived. 


\section{Survival Signature development and validation}

In the study, we set the TCGA cohort as train cohort, while ICGC and GSE57495 as test cohorts. All the analyses were performed in Rstudio software with implemented packages. All BCE genes were treated as continuous variable in signature development. Firstly, All BCE genes were verified in three GEO datasets by t.test between tumor and normal control samples. Secondly, BCE genes in TCGA were screened by univariate Cox analysis of overall survival (OS) with survival package. Finally, LASSO-penalized regression analysis for model construction with candidate genes with glmnet/Survivalpackage. After prognostic genes with altered expression in tumor were identified, the risk-score was calculated based on the formula: . In the equation, $n$ represented selected gene number, $\exp \left(\mathrm{G}_{\mathrm{i}}\right)$ represented the expression of gene $i$, while $\beta_{i}$ represented the coefficient for gene i. Based on the risk-score mean value, TCGA and ICGC patients were both stratified as high-risk and low-risk groups. For survival analysis, we applied KaplanMeier method to calculate the OS plot in different risk groups with survminer package. The log-rank test was performed to check the statistical significance. Forest plots were as draw to demonstrate the Hazard ratio $(\mathrm{HR})$ of selected prediction genes.

\section{Protein-protein interaction (PPI) analysis}

The PPI analysis of the prognostic genes was performed using the STRING database (http://stringdb.org), which provides critical assessment and integration of protein interactions.

\section{Immune infiltration analysis}

The immune infiltration score of immune cells between two groups were calculated with single-sample gene set enrichment analysis (ssGSEA) with gsva package.

\section{Cell lines}

The human pancreatic cancer cell lines (CFPAC-1, CAPAN-1, MIA PaCa-2, and PANC-1), hepatocellular carcinoma cell line HuH7, colorectal cancer cell line (HCT116 and SW480), and lung cancer cell line (A549) were purchased from Shanghai Institutes for Biological Sciences (Shanghai, China). The human Primary fibroblast N16 was denoted from Translational Medicine Research Center, Shanghai East Hospital. All cells were cultured in modified Eagle's medium (Gibco, Carlsbad, USA) supplemented with $10 \%$ fetal bovine serum, $1 \%$ penicillin, and $1 \%$ streptomycin at $37 \circ \mathrm{C}$ with $5 \% \mathrm{CO} 2$.

\section{Real-Time PCR}

Total RNA was isolated using TRIZOL Reagent (Invitrogen, Life Technologies) and was converted to cDNA using the PrimeScriptTM RT reagent Kit (Takara, Japan). Expression levels of mRNA were measured by real-time PCR (Applied Biosystems, 7500, USA) using SYBR Premix Ex TaqTM II (Takara, Japan). Total amount of mRNA was normalized to actin mRNA. The primer sequences were shown in

\section{Supplementary Table 2.}




\section{Immunohistochemical staining (IHC) with TMA}

A tissue microarray (Shanghai Outdo Biotech Co., Ltd. Shanghai, China) with 90 pancreatic carcinoma samples and paired adjacent samples were applied for IHC. Rabbit antihuman polyclonal ABAT antibody (Sigma-Aldrich, Cat\# HPA041690, USA) and BCAT2 antibody (Sigma-Aldrich, Cat\# HPA054091, USA) were used at a 1:500 dilution. Mouse antihuman CD68 antibody (Servicebio, Cat\# GB14043, China) was used at a 1:200 dilution. Quantitative analysis of the staining was performed with histochemistry score (Hscore), which was determined based on the intensity of staining and the proportion of labeled tumor cells as previously described.(12) The Hscore was calculated based on the formula: . In the equation, $i$ represented the graded of staining intensity, which included no staining $(i=0)$, weak $(i=1)$, moderate $(i=2)$, and strong ( $\mathrm{i}=3)$, while $p_{i}$ represented the percentage of labeled tumor cells with the corresponding stating intensity. To quantify the immune cell infiltration, positive cell in the tumoral and stromal compartments were enumerated separately and normalized per unit area as cells $/ \mathrm{mm}^{2}$.(13)

\section{Statistics.}

All analyses were set at two-sided $p$ value $<0.05$ as the threshold for statistical significance. The data were expressed as mean \pm standard deviation (SD).

\section{Result}

Six online open cohorts (i.e., TCGA, ICGC, GSE57495, GSE15471, GSE16515, and GSE28735) and one TMA cohort were included. Survival data were available for TCGA, ICGC, GSE57495, and TMA, which corresponding median survival times were 20.2, 19.1, 21.5, and 13.0 months, respectively. The detailed information is listed in Table 1. 
Table 1

Clinical parameters of included cohorts with survival data.

\begin{tabular}{|c|c|c|c|c|}
\hline Characteristics & $\begin{array}{l}\text { TCGA }(n= \\
178)\end{array}$ & $\begin{array}{l}\operatorname{ICGC}(n= \\
193)\end{array}$ & $\begin{array}{l}\text { GSE57495 }(n= \\
63)\end{array}$ & $\begin{array}{l}\text { Tissue microarray }(\mathrm{n}= \\
\text { 90) }\end{array}$ \\
\hline $\begin{array}{l}\text { Age (years, Mean } \pm \\
\text { SD) }\end{array}$ & $64.6 \pm 10.9$ & $55.1 \pm 25.3$ & NA & $61.3 \pm 10.8$ \\
\hline Gender (Male/Female) & $98 / 80$ & $106 / 86$ & NA & $57 / 33$ \\
\hline \multicolumn{5}{|l|}{ Location (n, \%) } \\
\hline Head & 138(77.5) & NA & NA & $51(56.7)$ \\
\hline Body/Tail & $29(16.3)$ & NA & NA & $31(34.4)$ \\
\hline Other & $11(6.2)$ & NA & NA & $8(8.9)$ \\
\hline \multicolumn{5}{|l|}{ Tumor grade $(n, \%)$} \\
\hline $\mathrm{G} 1+\mathrm{G} 2$ & 127(71.3) & $58(30.0)$ & NA & $57(63.3)$ \\
\hline $\mathrm{G} 3+\mathrm{G} 4$ & $49(27.5)$ & $32(16.6)$ & NA & $23(25.6)$ \\
\hline Unknown & $2(1.1)$ & $103(53.4)$ & NA & 10(11.1) \\
\hline \multicolumn{5}{|l|}{ Stage $(n, \%)$} \\
\hline$I+\|$ & $49(27.5)$ & $145(75.1)$ & $63(100)$ & $87(96.7)$ \\
\hline III + IV & $126(70.8)$ & $12(6.2)$ & $0(0)$ & $2(2.2)$ \\
\hline Unknown & $3(1.6)$ & $36(18.7)$ & $0(0)$ & $1(1.1)$ \\
\hline \multicolumn{5}{|l|}{ Survival status } \\
\hline Dead $(n, \%)$ & $92(51.7)$ & 148(76.7) & $42(66.7)$ & $59(65.6)$ \\
\hline Living $(\mathrm{n}, \%)$ & $86(48.3)$ & $45(23.3)$ & 21(33.3) & $31(34.4)$ \\
\hline $\begin{array}{l}\text { Median survival } \\
\text { (months) }\end{array}$ & 20.2 & 19.1 & 21.5 & 13.0 \\
\hline
\end{tabular}

Firstly, expression level of 48 BCE genes in pancreatic cancer were investigated in three GEO databases (GSE15471, GSE16515, and GSE28735). Six genes shown no alteration in any of GEO cohorts and were excluded (Fig. 1A). Univariate analysis was performed to screen survival associated BCE genes in TCGA, and eight genes (ABAT, ACAT1, BCAT1, BCAT2, DBT, HMGCLL1, IVD, and SDSL) were identified (Fig. 1B). Only SDSL showed no alteration in any of the three GEO cohorts and was excluded from further analysis. ABAT, ACAT1, and BCAT2 were significantly downregulated in pancreatic cancer in all three GEO cohorts. BCAT1, DBT, and HMGCLL1 were downregulated in at least one cohort. PPI analysis with the six BCE genes indicated that BCAT2 was the hub gene (Fig. 1C). 
Next, Lass-Cox analysis was applied to find out independent predictors for prognosis. Five of the six BCE genes were identified and the risk-score was calculated as follows: BCAT1×0.18 - ABAT×0.06 -

ACAT $1 \times 0.21-$ BCAT2 $\times 0.40+\mathrm{DBT} \times 0.13$. According to the risk-score, the high-risk and low-risk groups were stratified in TCGA, ICGC, and GSE57495 cohorts. The Kaplan-Meier plot indicated that high-risk group in TCGA had significantly worse prognosis than low-risk group $(p=0.02)$, with median survival times of 1.62 and 1.90 years, respectively (Fig. 2A). Moreover, the risk-score was validated using ICGC (high-risk vs. lowrisk: 1.25 years vs. 1.87 years; $p=0.01$, Fig. $2 B$ ) and GSE57495 (high-risk vs. low-risk: 1.49 years vs. 2.63 years; $p=0.02$, Fig. $2 C$ ). The association between risk groups and clinical parameters was shown in Table 2. The Kaplan-Meier plot of five BCE genes with the mean expression as cut-off value were shown in Fig. 2D. With the above survival analysis, we might find ABAT, ACAT1, and BCAT2 were favorable factors for a good prognosis in pancreatic cancer.

Table 2

Association between high-risk and low-risk groups with clinical parameters.

\begin{tabular}{|c|c|c|c|c|c|c|}
\hline \multirow[t]{2}{*}{ Characteristics } & \multicolumn{3}{|l|}{ TCGA cohort } & \multicolumn{3}{|l|}{ ICGC cohort } \\
\hline & $\begin{array}{l}\text { High-risk(n = } \\
98)\end{array}$ & $\begin{array}{l}\text { Low-risk }(n= \\
\text { 80) }\end{array}$ & $\begin{array}{l}P \\
\text { value }\end{array}$ & $\begin{array}{l}\text { High-risk(n = } \\
98)\end{array}$ & $\begin{array}{l}\text { Low-risk(n = } \\
95)\end{array}$ & $\begin{array}{l}P \\
\text { value }\end{array}$ \\
\hline Age $(n, \%)$ & & & 0.18 & & & 0.98 \\
\hline$\leq 65 y$ & $46(25.8)$ & $46(25.8)$ & & $38(19.7)$ & $40(20.7)$ & \\
\hline$>65 y$ & $51(28.7)$ & $34(19.1)$ & & $42(21.8)$ & $44(22.8)$ & \\
\hline Gender (n, \%) & & & 0.37 & & & 0.97 \\
\hline Female & $47(26.4)$ & $33(18.5)$ & & $44(22.8)$ & $42(21.8)$ & \\
\hline Male & $51(28.7)$ & $47(26.4)$ & & $54(27.8)$ & $52(26.9)$ & \\
\hline Location (n, \%) & & & 0.72 & & & \\
\hline Head & $75(42.1)$ & 63(35.4) & & - & - & \\
\hline Non-head & $23(12.9)$ & $17(9.6)$ & & - & - & \\
\hline $\begin{array}{l}\text { Tumor grade (n, } \\
\%)\end{array}$ & & & 0.73 & & & 0.55 \\
\hline $\mathrm{G} 1+\mathrm{G} 2$ & $69(38.8)$ & $58(32.6)$ & & $27(13.4)$ & $31(16.1)$ & \\
\hline $\mathrm{G} 3+\mathrm{G} 4$ & $28(15.7)$ & $21(11.8)$ & & $17(8.8)$ & $15(7.8)$ & \\
\hline Stage $(\mathrm{n}, \%)$ & & & 0.69 & & & 0.94 \\
\hline$I+I I$ & $26(14.6)$ & $23(12.9)$ & & $71(36.8)$ & $74(38.3)$ & \\
\hline III + IV & 71(39.9) & $55(30.9)$ & & $6(3.1)$ & $6(3.1)$ & \\
\hline
\end{tabular}


The predictive value of the risk-score was further investigated by considering the clinical parameters. In the TCGA cohort, the age, gender, AJCC stage, histological grade, tumor location, and risk-score were included in the survival analysis. Multivariate Cox analysis indicated that risk-score was an independent predictor for prognosis $(\mathrm{HR}=3.16,95 \% \mathrm{Cl}=1.63-6.12, \mathrm{p}<0.01 ; \mathrm{Fig} .3 \mathrm{~A})$. In ICGC, the age, gender, AJCC stage, and risk-score were included in the survival analysis. The results from the multivariate Cox analysis indicated that the risk-score was also an independent predictor for prognosis $(\mathrm{HR}=3.16,95 \% \mathrm{Cl}=1.04-$ $2.35, p=0.03$; Fig. 3B). The 5-gene risk-score showed robustness in the prognostic prediction of pancreatic cancer.

Expression of the five BCE genes were verified in several human cancer and normal cell lines by RT-PCR. As shown Fig. 4A, mRNA expression of ACAT1 and DBT was undetermined, while ABAT and BCAT2 were selected for further experiments due to the stable expression in all cancer cells. Consistently with expression level in three GEO cohorts, Hscore of ABAT and BCAT2 significantly downregulated in carcinoma than adjacent tissues by quantitative analysis of IHC staining on TMA (Fig. 4B). Tumors with T3 stage also shown lower Hscore of ABAT and BCAT2 than T1-2 stage (Fig. 4C). The Hscore of ABAT was also linearly correlated with BCAT2 $\left(R^{2}=0.875, p<0.001\right.$, Fig. 4D). Further, cases were divided into high-expression and low-expression groups, based on mean level of Hscore. As shown in Kaplan-Meier plot, both ABAT and BCAT2 with high-expression shown worse survival than low-expression cases (Fig. 4E).

Given the reported immunomodulatory function of BCAA, the immune infiltration scores were compared between high-risk with low-risk groups. As shown in Fig. 5, dendritic cells, macrophages, neutrophils, and Th2 cells were enriched in high-risk group both for TCGA cohort (Fig. 5A) and ICGC cohort (Fig. 5B). Here, we focused the function of macrophages in BCAA metabolism. To distinguish the exact location, CD68 ${ }^{+}$ total macrophage density was evaluated independently in TAM tumoral and stromal compartments. As shown in Fig. 5C, decreased positive cell density of stromal macrophage was observed in BCAT2 with low-expression than high-expression $(p<0.05)$, instead of tumoral macrophage. Besides, no significant association were detected for both tumoral and stromal macrophages with ABAT expression (Fig. 5D).

\section{Discussion}

In this study, BCAT1 and DBT were independent predictors for worse prognosis, while BCAT2, ACAT1, and ABAT were independent predictors for better prognosis. The BCAT enzymes, including BCAT1 and BCAT2, catalyzed the initial step of BCAA catabolism. Several studies have reported that BCAT1 promoted tumor proliferation and predicted poor survival in tumors, such as glioblastoma, gastric cancer, and non-small cell lung cancer.(14-16) Recent studies have shown that BCAT2-BCAA catabolism was critical in the development of pancreatic cancer, and the loss of BCAT2 expression significantly hampered the proliferation of this carcinoma.(17-19) A study on BCAA metabolism has indicated that pancreatic cancer showed decreased BCAA uptake, and BCAT1 and BCAT2 were not required for tumor formation. (16) In the present results using human pancreatic cancer sample, BCAT1 was positively associated with poor survival, but BCAT2, as the hub gene of the predictive model, showed otherwise. The ACAT1 enzyme 
acted in the degeneration of ketone, which is the last step in the catalysis of BCAA into cholesterol. Li et al. found that the upregulation of ACAT1 was positively correlated with the survival from pancreatic cancer.(20) In clear-cell renal-cell carcinoma, ACAT1 favored the survival prognosis, which was not consistent with the current data.(21) Such discrepancy between the animal experimental results with clinical findings indicated the BCAT1/2 and ACAT1 regulated the cancer in a tissue-specific manner. This result needed further investigation. DBT was one of the three enzymatic components of the branchedchain alpha keto dehydrogenase complex (BCKDH), which was also the key enzyme in BCAA metabolism. No study has directly investigated the function of DBT in tumors. However, the BCKDH-a was shown to sustain the growth of pancreatic cancer by regulating the lipid metabolism from BCAA.(17) Hence, the function of DBT should be further elucidated on the basis of the current finding.

Prospective studies have already validated the predictive value of serum BCAA for the risk of pancreatic carcinogenesis.(4-6) As described above, many enzymes for the metabolism of BCAA have been proved to be important in tumor progression and predictive for prognosis. This study was firstly focused on the immune infiltration of the BCE genes. The results showed that high-risk patient, who was determined by the 5-gene, harbored significantly enriched macrophage. The relation between BCAA metabolism with macrophage was poorly described. Theoretically, BCAA regulates the cell function not only by cellular signal transduction, similar to the mTOR pathway, but also triggers the metabolic reprogramming and cascades metabolite signal transduction. Ikeda et al. reported that BCAA sustained the balance of Treg cells by activating the mTOR pathway.(22) In glioblastoma, the phagocytic activity of macrophages was proved to be reduced by the tumor-excreted branched-chain ketoacids, the initial metabolites of BCAA catalyzed by BCAT1/2.(8) In addition, in macrophages, the metabolic profile was controlled by BCAT1 and associated with inflammatory conditions.(23) No studies have reported BCAA metabolism regulated macrophage functions in pancreatic cancer. Here, the current findings provided clues for the relation between BCAT2 and stromal macrophage infiltration. Whereas, further experiments to investigate the detailed mechanism were warranted.

In conclusion, a risk-score involving 5 BCE genes was proposed to predict the poor prognosis of pancreatic cancer in TCGA cohort, and the risk-score was validated in ICGC and GEO cohort. Based on the immune infiltration analysis, the underlying mechanism might be BCAT2 associated stromal macrophage infiltration.

\section{Declarations}

\section{Ethics approval and consent to participate}

This is a secondary analysis based on the open online database, and the informed consent was waived.

\section{Consent for publication}

Not applicable. 
Availability of data and materials

The datasets used and/or analyzed during the current study are available from the corresponding author on reasonable request.

\section{Competing interests}

The authors declare that they have no competing interests.

\section{Funding Support}

This work was supported by Shanghai Sailing Program (No. 21YF1408300).

\section{Authors' contributions}

CK, JZT, and ZJJ designed and supervised the project. YXJ and CK performed the clinical procedure. JZT, ZJJ, and LJQ collected the samples and performed the analysis and interpreted data. JZT and ZJJ drafted the manuscript. CK revised the manuscript for important content.

\section{Acknowledgements}

Not applicable.

\section{References}

1. Siegel RL, Miller KD, Fuchs HE, Jemal A. Cancer Statistics. 2021. CA: a cancer journal for clinicians. 2021;71(1):7-33.

2. Pavlova NN, Thompson CB. The Emerging Hallmarks of Cancer Metabolism. Cell Metabol. 2016;23(1):27-47.

3. Ananieva EA, Wilkinson AC. Branched-chain amino acid metabolism in cancer. Curr Opin Clin Nutr Metab Care. 2018;21(1):64-70.

4. Mayers JR, Wu C, Clish CB, Kraft P, Torrence ME, Fiske BP, et al. Elevation of circulating branchedchain amino acids is an early event in human pancreatic adenocarcinoma development. Nature medicine. 2014;20(10):1193-8.

5. Katagiri R, Goto A, Nakagawa T, Nishiumi S, Kobayashi T, Hidaka A, et al. Increased Levels of Branched-Chain Amino Acid Associated With Increased Risk of Pancreatic Cancer in a Prospective Case-Control Study of a Large Cohort. Gastroenterology. 2018;155(5):1474-82 e1.

6. Zhang S, Zeng X, Ren M, Mao X, Qiao S. Novel metabolic and physiological functions of branched chain amino acids: a review. J Anim Sci Biotechnol. 2017;8:10.

7. Poh AR, Ernst M. Tumor-Associated Macrophages in Pancreatic Ductal Adenocarcinoma: Therapeutic Opportunities and Clinical Challenges. Cancers. 2021;13(12). 
8. Silva LS, Poschet G, Nonnenmacher Y, Becker HM, Sapcariu S, Gaupel AC, et al. Branched-chain ketoacids secreted by glioblastoma cells via MCT1 modulate macrophage phenotype. EMBO Rep. 2017;18(12):2172-85.

9. Ko JH, Olona A, Papathanassiu AE, Buang N, Park KS, Costa ASH, et al. BCAT1 affects mitochondrial metabolism independently of leucine transamination in activated human macrophages. Journal of cell science. 2020;133(22).

10. Ericksen RE, Lim SL, McDonnell E, Shuen WH, Vadiveloo M, White PJ, et al. Loss of BCAA Catabolism during Carcinogenesis Enhances mTORC1 Activity and Promotes Tumor Development and Progression. Cell Metabol. 2019;29(5):1151-65. e6.

11. Lamb J, Crawford ED, Peck D, Modell JW, Blat IC, Wrobel MJ, et al. The Connectivity Map: using geneexpression signatures to connect small molecules, genes, and disease. Science. 2006;313(5795):1929-35.

12. Detre S, Saclani Jotti G, Dowsett M. A "quickscore" method for immunohistochemical semiquantitation: validation for oestrogen receptor in breast carcinomas. J Clin Pathol. 1995;48(9):876-8.

13. Sadozai H, Acharjee A, Gruber T, Gloor B, Karamitopoulou E. Pancreatic Cancers with High Grade Tumor Budding Exhibit Hallmarks of Diminished Anti-Tumor Immunity. Cancers. 2021;13(5).

14. Tonjes M, Barbus S, Park YJ, Wang W, Schlotter M, Lindroth AM, et al. BCAT1 promotes cell proliferation through amino acid catabolism in gliomas carrying wild-type IDH1. Nature medicine. 2013;19(7):901-8.

15. Xu Y, Yu W, Yang T, Zhang M, Liang C, Cai X, et al. Overexpression of BCAT1 is a prognostic marker in gastric cancer. Human pathology. 2018;75:41-6.

16. Mayers JR, Torrence ME, Danai LV, Papagiannakopoulos T, Davidson SM, Bauer MR, et al. Tissue of origin dictates branched-chain amino acid metabolism in mutant Kras-driven cancers. Science. 2016;353(6304):1161-5.

17. Lee JH, Cho YR, Kim JH, Kim J, Nam HY, Kim SW, et al. Branched-chain amino acids sustain pancreatic cancer growth by regulating lipid metabolism. Exp Mol Med. 2019;51(11):1-11.

18. Li JT, Yin M, Wang D, Wang J, Lei MZ, Zhang Y, et al. BCAT2-mediated BCAA catabolism is critical for development of pancreatic ductal adenocarcinoma. Nat Cell Biol. 2020;22(2):167-74.

19. Dey P, Baddour J, Muller F, Wu CC, Wang H, Liao WT, et al. Genomic deletion of malic enzyme 2 confers collateral lethality in pancreatic cancer. Nature. 2017;542(7639):119-23.

20. Li J, Gu D, Lee SS, Song B, Bandyopadhyay S, Chen S, et al. Abrogating cholesterol esterification suppresses growth and metastasis of pancreatic cancer. Oncogene. 2016;35(50):6378-88.

21. Chen L, Peng T, Luo Y, Zhou F, Wang G, Qian K, et al. ACAT1 and Metabolism-Related Pathways Are Essential for the Progression of Clear Cell Renal Cell Carcinoma (ccRCC), as Determined by Coexpression Network Analysis. Frontiers in oncology. 2019;9:957.

22. Ikeda $K$, Kinoshita M, Kayama H, Nagamori S, Kongpracha P, Umemoto E, et al. Slc3a2 Mediates Branched-Chain Amino-Acid-Dependent Maintenance of Regulatory T Cells. Cell reports. 
2017;21(7):1824-38.

23. Papathanassiu AE, Ko JH, Imprialou M, Bagnati M, Srivastava PK, Vu HA, et al. BCAT1 controls metabolic reprogramming in activated human macrophages and is associated with inflammatory diseases. Nature communications. 2017;8:16040.

\section{Figures}

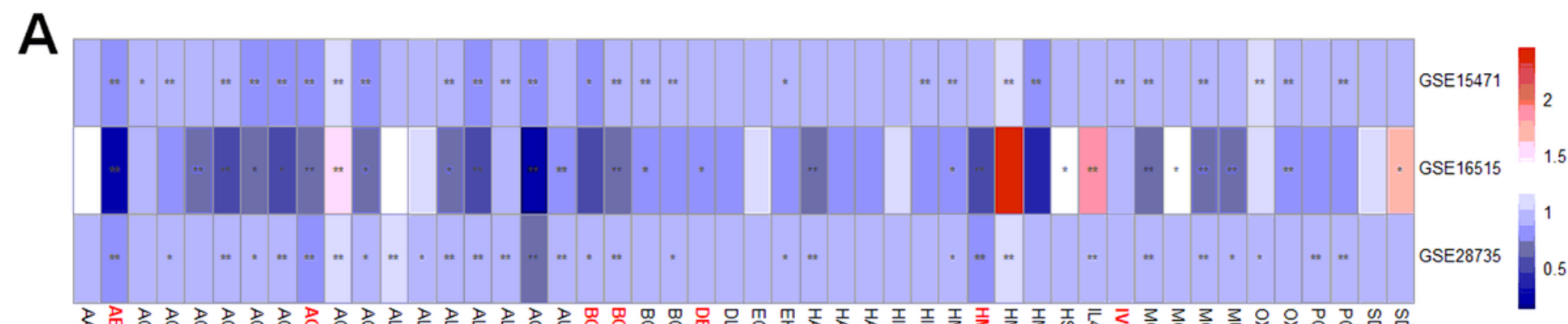

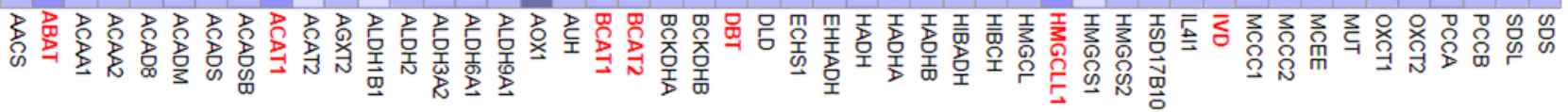

B

$\begin{array}{lrcc} & \text { P-value } & \text { Hazard ratio } \\ \text { ABAT } & 0.016 & 0.757(0.603-0.950) \\ \text { ACAT1 } & 0.013 & 0.620(0.426-0.903) \\ \text { BCAT1 } & <0.001 & 1.457(1.166-1.820) \\ \text { BCAT2 } & <0.001 & 0.466(0.308-0.703) \\ \text { DBT } & 0.032 & 2.051(1.062-3.961) \\ \text { HMGCLL1 } & 0.026 & 0.626(0.415-0.945) \\ \text { IVD } & 0.044 & 0.637(0.410-0.988) \\ \text { SDSL } & 0.003 & 0.582(0.409-0.827) & \\ & & & 0\end{array}$

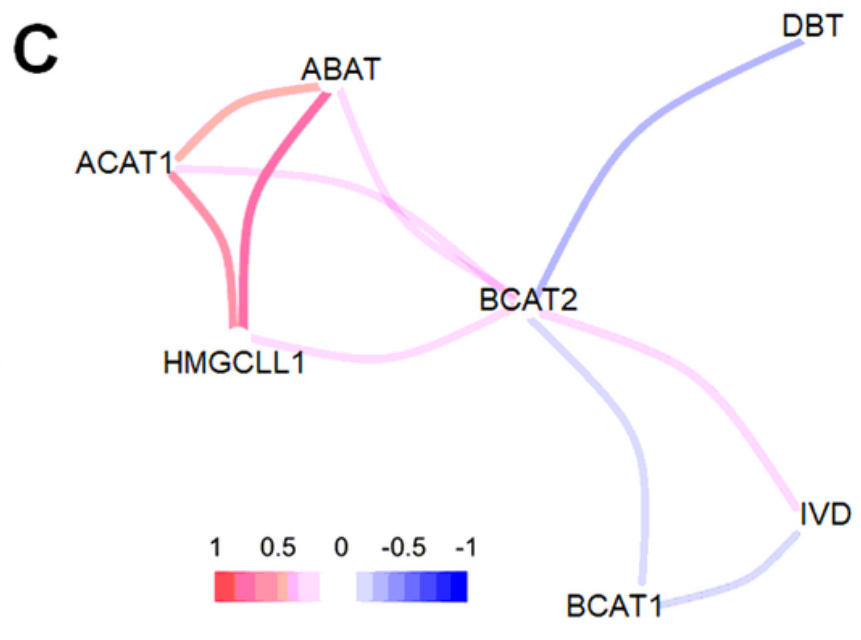

Figure 1

Identification of the prognosis associated BCE genes for pancreatic cancer. (A) Heatmap depicted all BCE genes expression level between carcinoma and adjacent tissue in $3 \mathrm{GEO}$ databases. Legend shows the fold change. Star in the box indicated significant $p$-value $\left({ }^{*}, p<0.05 ;{ }^{*}, p<0.01\right)$. (B) the univariate Cox analysis with TCGA data identified eight BCE genes as shown in the Forest plot. C. PPI network shown the correlation network of BCE genes, and BCAT2 was the hub gene. 
A

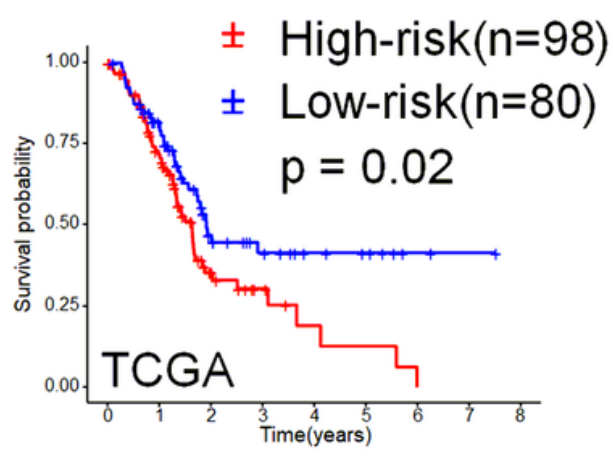

B

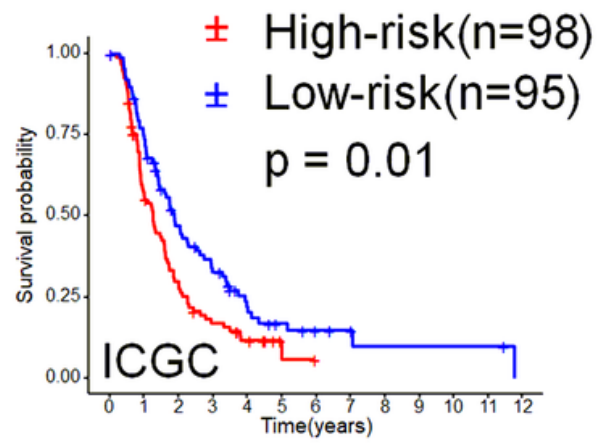

C

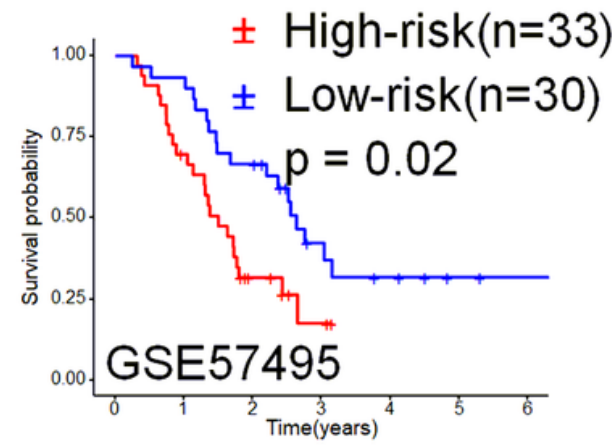

D
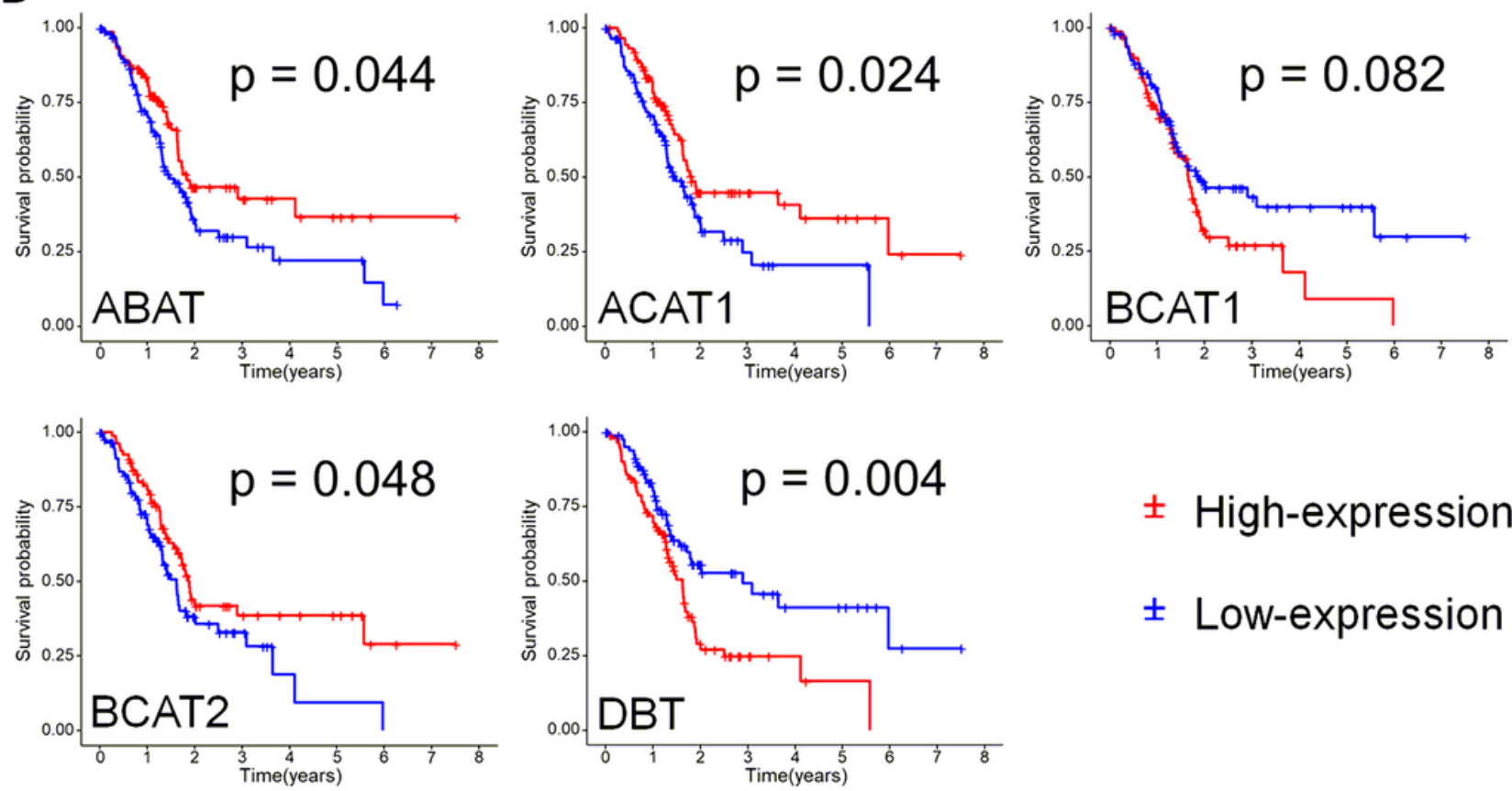

Figure 2

Prognostic analysis of the 5-BCE genes signature. (A-C) Kaplan-Meier plots shown survival between highrisk group and low-risk group in TCGA cohort (A), ICGC cohort (B), and GSE57495 cohort (C). (D) KaplanMeier plots of five BCE genes was shown. 


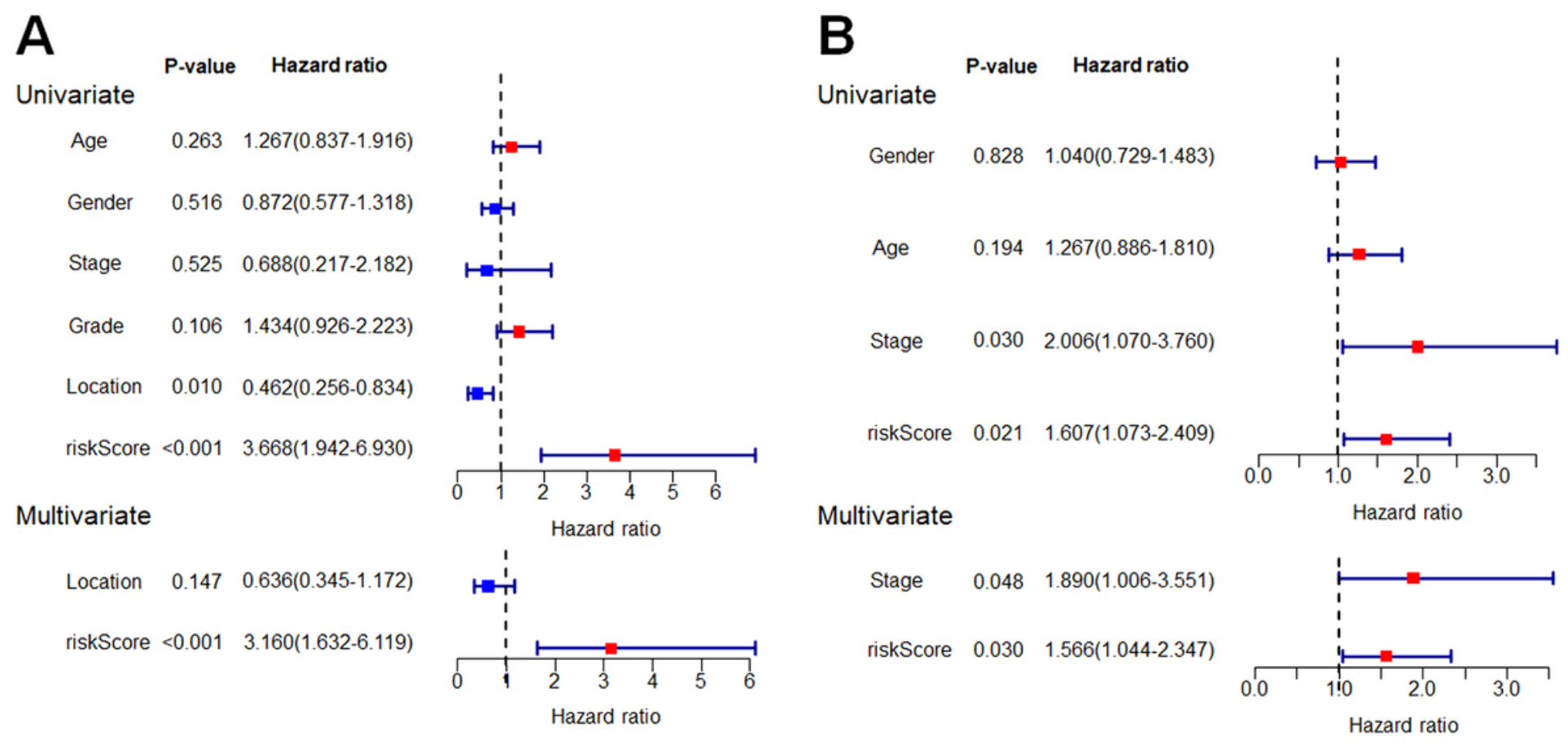

\section{Figure 3}

Results of the univariate and multivariate Cox regression analyses regarding OS in the TCGA cohort (A) and ICGC cohort (B). 
A

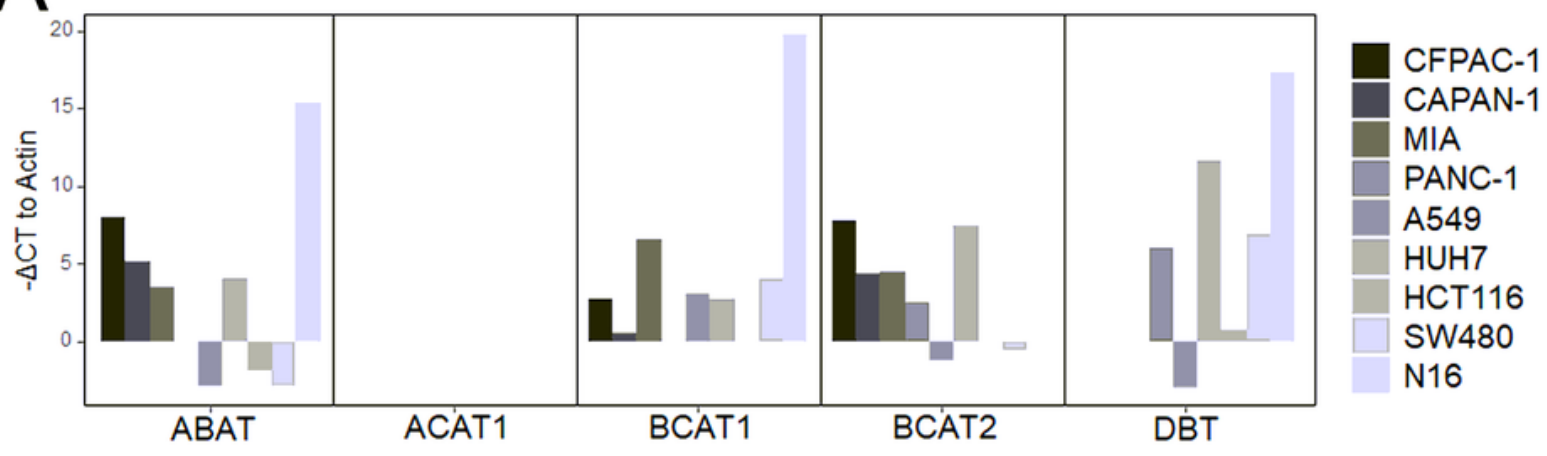

B

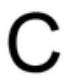

D
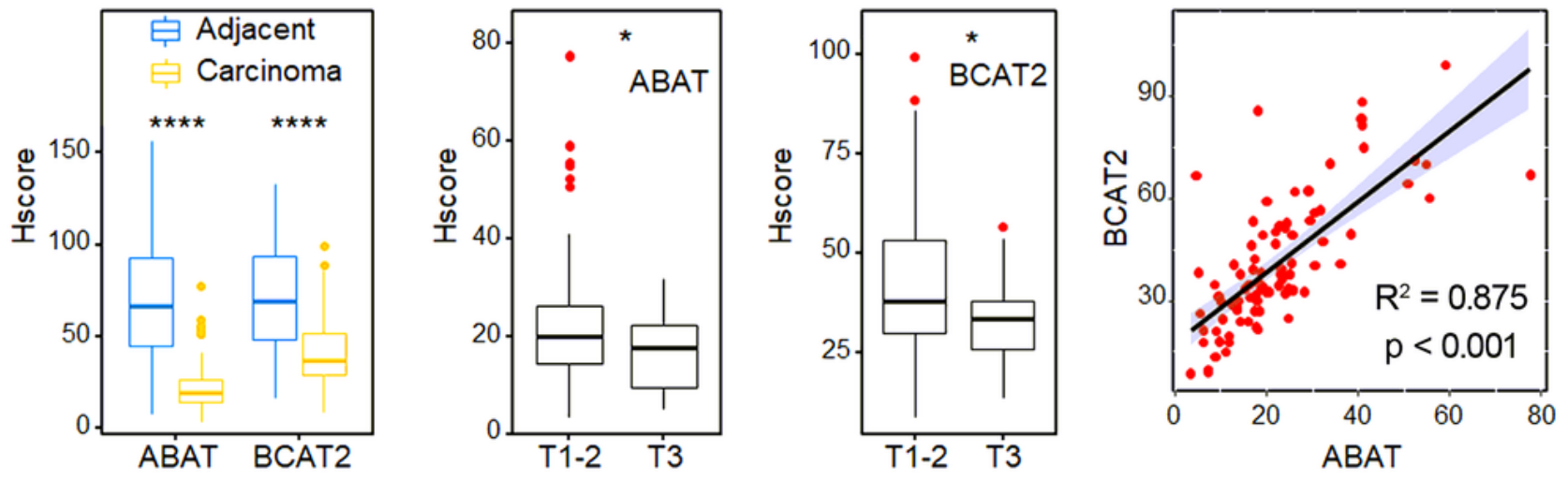

E

- High-expression - Low-expression
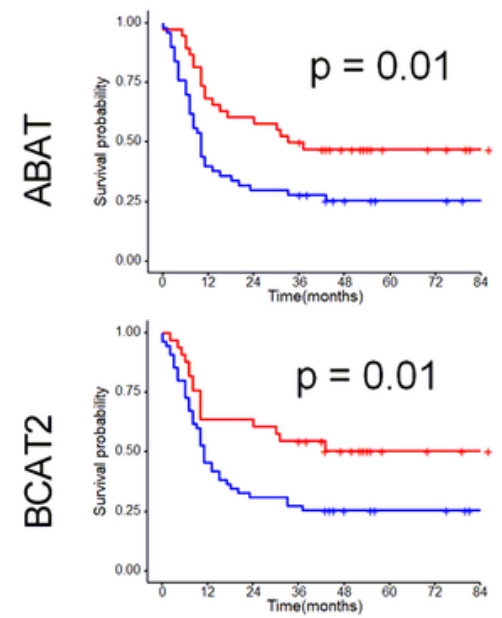

High-expression

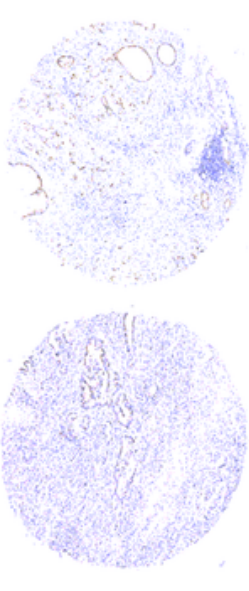

Carcinoma
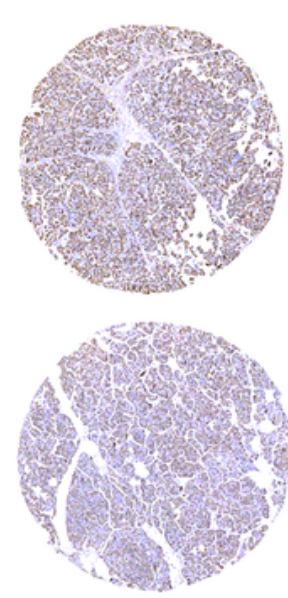

Adjacent
Low-expression

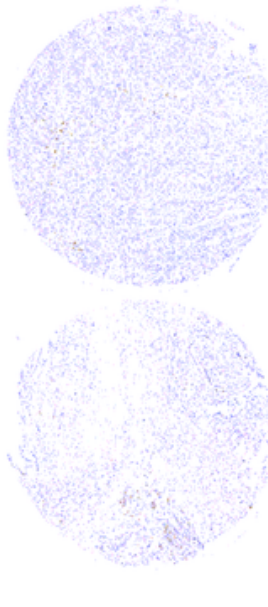

Carcinoma
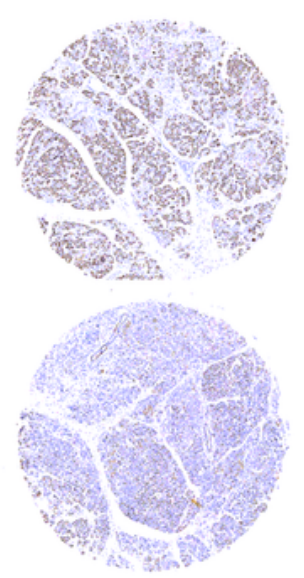

Adjacent

\section{Figure 4}

BCE gene verification in cell lines and TMA. (A). RT-PCR shown the five BCE genes mRNA expression in different cancer cell lines and normal human cell line. (B) Hscore of ABAT and BCAT2 between carcinoma

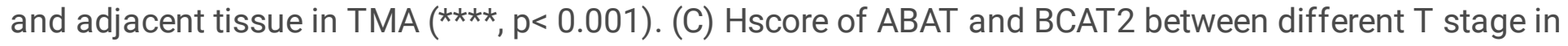
TMA (*, p 0.05$)$. (D) correlation plot between ABAT and BCALT2. (E) Hscore of ABAT and BACT2 were associated with pancreatic cancer OS and the representative cases was presented. 
A

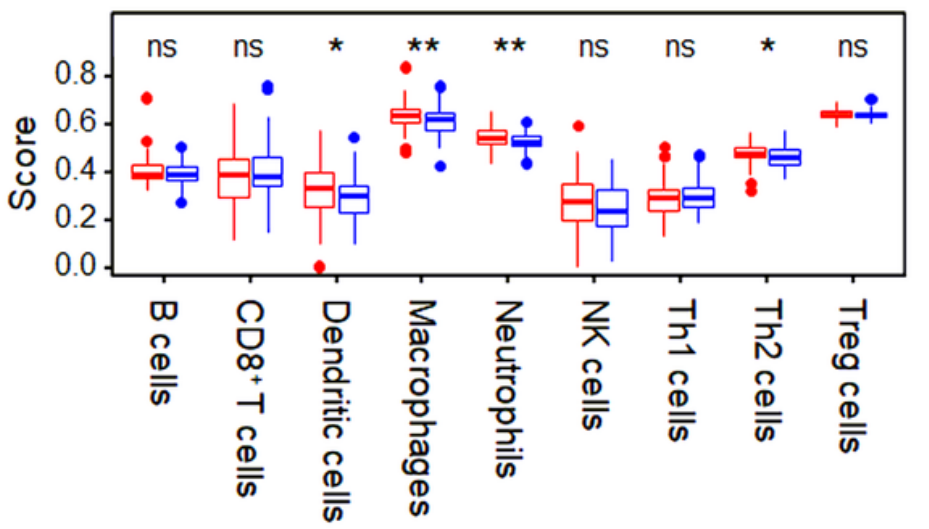

B 追High-risk 追Low-risk

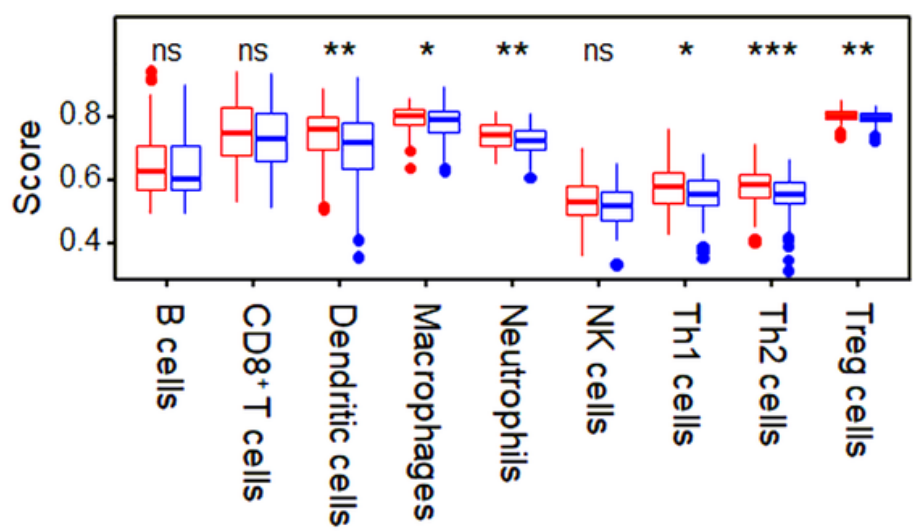

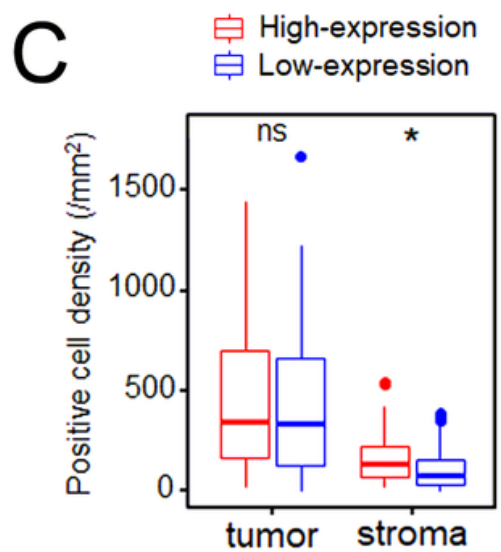
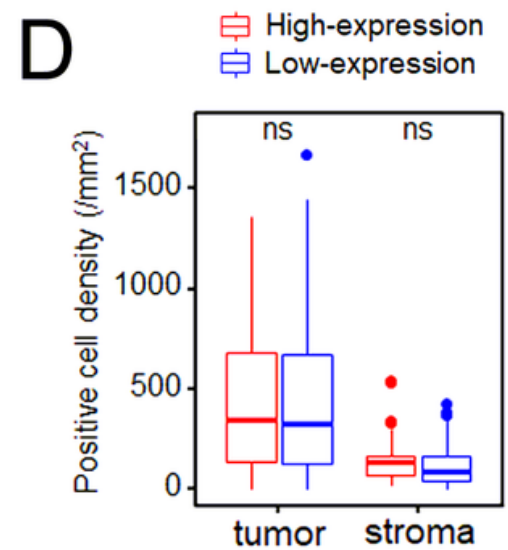

Figure 5

Immune infiltration in pancreatic cancer. The immune infiltration scores were compared between high-risk with low-risk groups for TCGA cohort (A) and ICHC cohort (B). Positive cell density of CD 68+ macrophage in TAM between different BCAT2 (C) and ABAT (D) expression level were detected, respectively. ${ }^{*}, \mathrm{p}<0.05$; $\star *, p<0.01 ; * \star *, p<0.005)$

\section{Supplementary Files}

This is a list of supplementary files associated with this preprint. Click to download.

- SupplementalTable1.xlsx

- SupplementalTable2.xlsx 